\title{
Traduire, lire Claude Simon
}

\section{Martine Créac'H}

\section{OpenEdition}

\section{Journals}

Édition électronique

URL : http://journals.openedition.org/ccs/938

DOI : $10.4000 /$ ccs. 938

ISSN : 2558-782X

\section{Éditeur :}

Presses universitaires de Rennes, Association des lecteurs de Claude Simon

\section{Édition imprimée}

Date de publication : 30 avril 2015

Pagination : 15-18

ISBN : 9782753539990

ISSN : 1774-9425

Référence électronique

Martine Créac'H, «Traduire, lire Claude Simon », Cahiers Claude Simon [En ligne], 10 | 2015, mis en ligne le 22 septembre 2017, consulté le 01 mai 2019. URL : http://journals.openedition.org/ccs/938 ; DOI : $10.4000 /$ ccs.938 
cessus en cours. Il reste que si nous admettons que le "traduire " peut aussi désigner les phénomènes de translation mettant en jeu le champ complexe des paramètres d'une langue, nous sommes cette fois au plus près du projet d'un écrivain qui a souhaité s'en remettre aux " transferts et transports de sens " ${ }^{7}$ actifs dans la langue, aux " harmoniques, [...] dissonances, passages, dérapages, contrastes etc. ${ }^{8}$, les accepter et les conduire tout à la fois: procéder à un " autrement dit " ${ }^{9}$ qui est un " autrement fait ".

C'est peut-être pour cette raison que le dossier que nous présentons nous mène au cœur de questions qui sont aussi des interrogations critiques de première importance déjà rencontrées dans nos analyses des textes de Simon: la valeur fondatrice de l'incipit, la part dialogique des textes, la trace de l'oral dans l'écrit, l'exploitation des clichés, les réseaux d'images, le narratif et le descriptif, les vocabulaires techniques, le glissement du plan de la réalité à celui de sa représentation, le dire du chaos, l'expression de la simultanéité, la rêverie de l'originel, l'intraduisible et l'indicible.

Le principe d'une énonciation redoublée est fréquent chez Claude Simon: un narrateur rapporte les propos d'un second personnage-narrateur, non sans assumer le plus souvent le risque de l'approximation. Qu'en résulte-t-il? Un énoncé troublé pour le lecteur? Non, plutôt une séquence où tout fait signe en direction du fonctionnement du texte dans sa globalité: les butées lexicales, décrochements syntaxiques, insistances tropiques, récurrences métaphoriques, déports sur les effets de contexte éclairants... Il ne semble donc pas inopportun d'espérer de cette enquête dans le champ du " traduire Claude Simon " un surcroît d'attention à ce qui, dans l'œuvre, subsiste de son faire et assure par là-même la potentialité renouvelable de ses lectures.

Anne-Yvonne JULIEN

Université de Poitiers

\section{TRADUIRE, LIRE CLAUDE SIMON}

Le titre de ce recueil reprend le titre d'une journée d'étude qui eut lieu le $1^{\text {er juin }} 2013$ à l'université Paris 8. Autour des traductions des romans de Claude Simon conservées à la bibliothèque de Paris 8 et présentées dans ce volume par Fanny Lambert, cette journée avait été conçue comme un atelier de traduction. Après une matinée consacrée aux traductions en Europe du

7. Claude Simon, Discours de Stockholm, op. cit., p. 28. Voir l'ouvrage collectif Transports, les métaphores de Claude Simon, dir. I. Albers et W. Nitsch, Frankfurt, éd. Peter Lang, 2006.

8. Claude Simon, «L'absente de tous bouquets", Quatre conférences, éd. Minuit, 2012, p. 59.

9. Pour reprendre l'expression de Paul Ricœur lorsqu'il évoque «la traduction à l'intérieur de la même communauté langagière ", Sur la traduction, op. cit., p. 43-45. 
Nord et aux particularités de la langue simonienne et avant l'analyse d'une expérience pédagogique à partir du Tramway, la table ronde de l'après-midi, préparée par Marie-Jeanne Zenetti ${ }^{10}$ et ses étudiants, a réuni chercheurs et traducteurs autour de la première page de L'Herbe dans des versions en langues allemande, anglaise, chinoise, espagnole, géorgienne et russe. Nous avons souhaité garder cette dimension active et expérimentale dans le présent volume en sollicitant, à côté du témoignage de 1985 de C. G. Bjürstrom présenté par Alastair Duncan, celui d'Eva Moldenhauer qui, en 2015, parle de son expérience de traductrice (de La Corde raide en 1964 aux Quatre conférences en 2014) dans un texte intitulé: "Dans l'atelier du traducteur ". Nous avons publié la traduction originale et inédite du début de L'Herbe par Peter France, ouvert l'atelier aux langues danoise et italienne et présenté plusieurs avanttextes de traducteurs, manuscrits ou tapuscrits qui donnent à " voir les mots " selon le vœu de Simon dans la préface d'Orion aveugle, rappellent son intérêt pour la "page d'écriture " mais suggèrent aussi, par les ratures et les repentirs autour des traductions publiées, d'autres textes possibles.

À partir de ces pratiques très concrètes s'esquissent des réflexions plus générales que nous avons choisi de mettre en valeur dans l'organisation du recueil. La première partie définit la singularité de l'œuvre de Claude Simon et les défis qu'elle pose pour la traduction. Karin Holter, qui a traduit en norvégien L'Acacia et Le Jardin des Plantes, montre que ces défis surgissent dès la lecture des premières lignes du roman qui posent les questions premières du mouvement du texte, de son rythme, de la voix, de la force génératrice des mots. David Zemmour analyse la spécificité du style simonien et sa paradoxale étrangeté au cœur même de la langue française.

Un second ensemble expose les résistances des différents contextes culturels et linguistiques. Par la comparaison de plusieurs traductions de L'Herbe en langue anglaise, Peter France montre les dangers de l'adaptation aux normes de la culture du lecteur. Pour ce spécialiste de la traduction littéraire, celle-ci ne peut éviter le "dépaysement " et peut prendre le risque de faire "revivre les personnages de Simon dans un monde qu'ils n'avaient jamais connu». À partir d'exemples très précis, en particulier le choix d'adapter ou non les prénoms ou les noms propres, Manuel Tost Planet compare deux versions espagnoles de L'Herbe, celles des écrivains Miguel Angel Asturias et Esther Tusquets. À propos de la traduction en géorgien de La Route des Flandres, Gaston Bouatchidzé étudie notamment les problèmes posés par les clichés

10. Qu'elle soit remerciée vivement pour sa participation et pour l'aide apportée à l'organisation de cette journée. Celle-ci a pu avoir lieu grâce au soutien financier de l'université Paris 8 et, notamment, de Carole Letrouit directrice de la bibliothèque, de Pierre Bayard et Mireille Séguy directeurs de l'Équipe de recherche "Littérature et histoires " ainsi que de l'Institut français. Je remercie aussi chaleureusement Fanny Lambert et Thierry Kiefer qui nous ont aidés à préparer cette journée. 
de langue. Enfin, la contribution proposée par Metka Zupančič et Mojca Schlamberger Brezar rend compte d'une très intéressante expérience pédagogique: la comparaison des traductions d'un même extrait du Tramway par des étudiants de l'université de Ljubljana en Slovénie et des étudiants de l'université d'Alabama aux États-Unis.

Faut-il choisir la langue cible contre la langue source? Jufang Jin, qui vient de traduire en chinois L'Acacia de Claude Simon après avoir soutenu un doctorat sur les rapports entre l'Histoire et la fiction, suggère que la traduction des romans de Claude Simon obéit à une "pulsion-de-traduction ", pour reprendre l'expression qu'elle emprunte à Antoine Berman: le travail de Claude Simon sur la mémoire, celle des guerres notamment, rend nécessaire la traduction de ses romans pour " tous les peuples qui ont traversé les vicissitudes du $\mathrm{xx}^{\mathrm{e}}$ siècle ». Elle s'emploie donc à ouvrir l'accès de son œuvre au public chinois. Assumant le parti pris d'une traduction philologique, le chercheur russe Alexey Vishnyakov, qui prépare actuellement une édition critique en russe de L'Herbe et de L'Invitation, veut au contraire souligner le caractère étranger du texte original dans la langue d'accueil, notamment par l'emploi de notes. Étudiant les versions danoises du Jardin des Plantes et du Tramway, Hans Peter Lund propose la notion de langue-texte pour désigner «la langue caractéristique réalisée spécifiquement par un texte baignant dans un domaine linguistique (syntaxe, grammaire) et un champ culturel (vocabulaire, références) particuliers ».

Loin d'être une simple transcription, la traduction est bien une écriture, un "cheminement" dans la langue, comme le montrent également Irene Albers et Johan Härnsten à partir de grandes traductions historiques. Pour Irene Albers qui étudie les traductions en langue allemande, l'analyse des retraductions et des variantes des avant-textes d'Erika et Elmar Tophoven souligne le "processus de la traduction " qui envisage le texte de Simon non comme un "objet déterminé et clos" mais comme un "événement ». À partir de la correspondance entre Claude Simon et son traducteur suédois C. G. Bjurström, Johan Härnsten souligne également, avec Henri Meschonnic $^{11}$, la dimension dynamique du traduire: "élaboration d'un original qui, en toute rigueur, n'existait pas avant l'acte de traduction ", travail d'artisan sur une matière-langue comparable au travail de l'écrivain.

La dernière partie est consacrée aux nouvelles traductions des romans de Simon. Elles sont autant de lectures nouvelles ouvertes parfois à des significations que l'auteur lui-même n'avait pas prévues. Étudiant les traductions italiennes des Géorgiques, Emilia Surmonte note que le projet d'écriture de Simon "s'avère aujourd'hui beaucoup plus proche des modalités de lecture 
e-textuelle - dialogique et plurilogique -, que de celles de son époque". Wolfram Nitsch analyse la façon dont l'histoire des traductions en Allemagne a orienté les lectures de l'œuvre. Celles d'Eva Moldenhauer, qui font de Claude Simon un " ethnographe du monde moderne ", s'écartent à la fois des théories du Nouveau Roman et des premières traductions de l'œuvre en allemand. Parce qu'elles révèlent l'incomplétude de la lecture, font du signifié « l'effet du signifiant ${ }^{12}$ ", ces multiples expériences de traductions ouvrent sur un futur de l'œuvre de Claude Simon, à des "images ultérieures " qu'interrogera également, dans le même volume, l'écrivain Marcel Beyer dans un texte intitulé «Feuille, baraque, écorce, bordel. Claude Simon à Mühlberg sur Elbe ».

Ce recueil, qui propose de nouvelles façons de lire l'œuvre de Claude Simon, ouvre aussi de nouveaux chantiers d'étude. À partir d'exemples de co-traductions de L'Herbe (en allemand par Erika et Elmar Tophoven, en espagnol par Miguel Angel Asturias et Blanca de Asturias, par Esteban Busquets et Esther Tusquets), il interroge le statut de l'auteur. Analysant l'évolution en un demi-siècle des politiques éditoriales, il est aussi une passionnante contribution à l'étude de la circulation des œuvres littéraires entreprise dans un récent ouvrage dirigé par Gisèle Sapiro ${ }^{13}$. Comparant les anglais des deux côtés de l'Atlantique, le bokmål et le landsmål en Norvège, les espagnols d'Amérique et de la péninsule ibérique, ces multiples contributions nous invitent enfin à lire les oeuvres de Claude Simon non seulement dans l'écart entre l'original et la traduction mais aussi «entre les langues ${ }^{14}$ ».

Martine CRÉAC'H

Université Paris 8

12. Jacques Derrida, Le Monolinguisme de l'autre ou la prothèse d'origine, Paris, Galilée, 1996, p. 18.

13. Translatio. Le marché de la traduction en France à l'heure de la mondialisation, Gisèle Sapiro dir., Paris, CNRS éditions, 2008.

14. J'emprunte l'expression à Barbara Cassin, "L'Énergie des intraduisibles. La traduction comme paradigme pour des sciences humaines " in Philosopher en langues. Les intraduisibles de la traduction, B. Cassin dir., Paris, Éditions Rue d’Ulm, Presses de l'École normale supérieure, 2014, p. 17. 